\title{
Endoscopic submucosal dissection: How to be more efficient?
}

\section{(ㄷ)(1) $\odot$}

Authors

Thomas Lambin ${ }^{1,2}$, Jérôme Rivory ${ }^{1}$, Timothée Wallenhorst ${ }^{3}$, Romain Legros ${ }^{4}$, Frédéric Monzy , Jérémie Jacques $^{4}$, Mathieu Pioche ${ }^{1,2}$

Institutions

1 Gastroenterology and Endoscopy Unit, Pavilion L, Edouard Herriot Hospital, Lyon, France

2 Inserm U1032, Labtau, Lyon, France

3 Department of Gastroenterology, Pontchaillou University Hospital, Rennes, France

4 Gastroenterology and Endoscopy Unit, Dupuytren university Hospital, Limoges, France

5 Clinique Claude Bernard, Albi, France

submitted 1.4 .2021

accepted after revision 13.7.2021

Bibliography

Endosc Int Open 2021; 9: E1720-E1730

DOI 10.1055/a-1554-3884

ISSN 2364-3722

(C) 2021. The Author(s).

This is an open access article published by Thieme under the terms of the Creative Commons Attribution-NonDerivative-NonCommercial License, permitting copying and reproduction so long as the original work is given appropriate credit. Contents may not be used for commercial purposes, or adapted, remixed, transformed or built upon. (https://creativecommons.org/licenses/by-nc-nd/4.0/)

Georg Thieme Verlag KG, Rüdigerstraße 14,

70469 Stuttgart, Germany

Corresponding author

Dr. Thomas Lambin, Endoscopy unit - Digestive Disease

department, Pavilion L - Edouard Herriot Hospital, 69437

Lyon Cedex, France

Fax: +0320445564

thomaslambin@hotmail.fr

\section{ABSTRACT}

Endoscopic submucosal dissection (ESD) allows an "en bloc" resection with safety margins (R0 resection) regardless of the size of the lesion. However, while Ro brings a real benefit for the patient, it is not considered sufficient by many experts to justify the technical difficulties and the longer procedure time compared to piecemeal mucosectomy. The aims of this review are to provide several technical and strategical tips to help you save time and become comfortable during ESD procedures. ESD is divided into several intertwined phases: injection, incision, access to the submucosae, and submucosal dissection itself. During injection there are some mistakes that should not be made: a superficial injection, or on the contrary, a too deep injection. A good needle and good injection technique are mandatory. Some techniques, such as repeated injection or prolonged lifting solution, can help maintain the lift. After this step, mucosal incision can be made, taking care to have a good margin to allow an R0 resection. Starting the mucosal incision from a small point allows calibration of the depth of the incision and then obtaining a nice incision. Trimming is also very important to widen submucosal access. Then comes the submucosal dissection itself. Strategies such as the tunnel strategy or the pocket creation method can help to facilitate dissection, but more importantly, traction systems have become unavoidable, especially in the stomach and colon. Most common complications are bleeding and perforation, and they usually can be managed endoscopically.
Endoscopic submucosal dissection (ESD) is a fascinating technique which allows an "en bloc" resection with safety margins ( $\mathrm{R} 0$ resection) regardless of the size of the lesion. This $\mathrm{R} 0$ quality level enables pathologists to analyze the specimen optimally and to diagnose possible lymphovascular invasion or budding, or even to measure the exact depth of parietal invasion. However, while R0 brings a real benefit for the patient, this benefit is not considered sufficient by many experts to justify the technical difficulties and the longer procedure time compared to piecemeal mucosectomy [1]. However, modern dissection with all the technical and strategical advances that have appeared in recent years no longer resembles the original procedures. The aims of this review are to provide several technical and strategical tips to help you save time and become comfortable during ESD procedures.

After characterization of the lesion, the submucosal dissection procedure is divided into several intertwined phases: injection, incision, access to the submucosa, and submucosal dissection itself ( $\mathbf{F i g} \mathbf{1} \mathbf{1}$ ). For each step, we will outline the technical issue and how to fix it in terms of material, strategies, and 

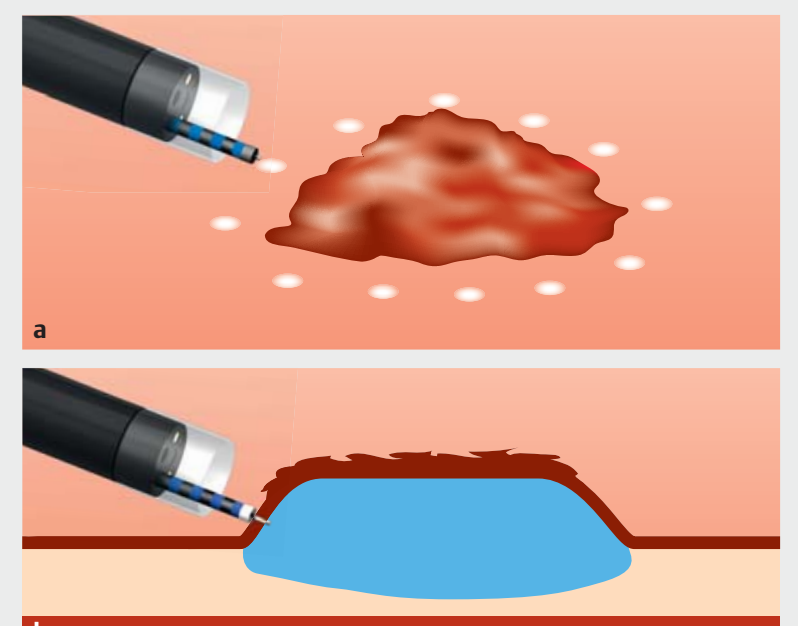

b
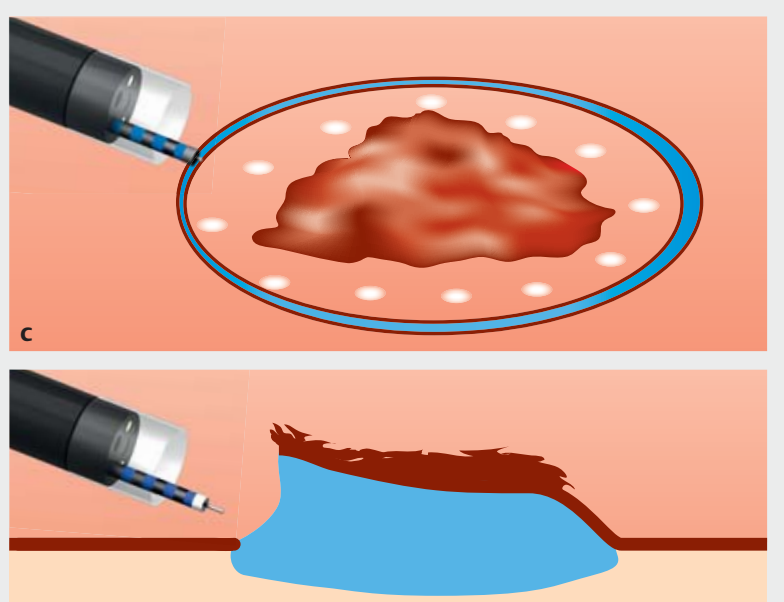

d

- Fig. 1 Steps in the ESD procedure. a Marking the lesion (optional in the colon). b Submucosal injection. c Circumferential mucosal incision (original techniques). d Submucosal dissection.

technical tips. In all cases, a transparent cap placed at the tip of the endoscope is essential to avoid being too close to the working area and to keep a free field of view. To place it, the overpressure evacuation hole (initially created to avoid gas emboli) must be placed at the 12 o'clock position, at a distance from the suction port so as not to suck the mucosa through this hole. Also, $\mathrm{CO}_{2}$ insufflation is mandatory because it has been associated with lower pain and lower rate of adverse events (AEs) [2-4]. The key steps of the procedure are described below.

\section{Essential prerequisite for colonic ESD: colonoscopy without loops}

This is probably the pivotal moment. Indeed, it is only possible to work well when the movements of the endoscope are free and there is a minimum response to the movements imposed on the handle. This is one of the elements that makes colonic dissection much more difficult than rectal or gastric dissection

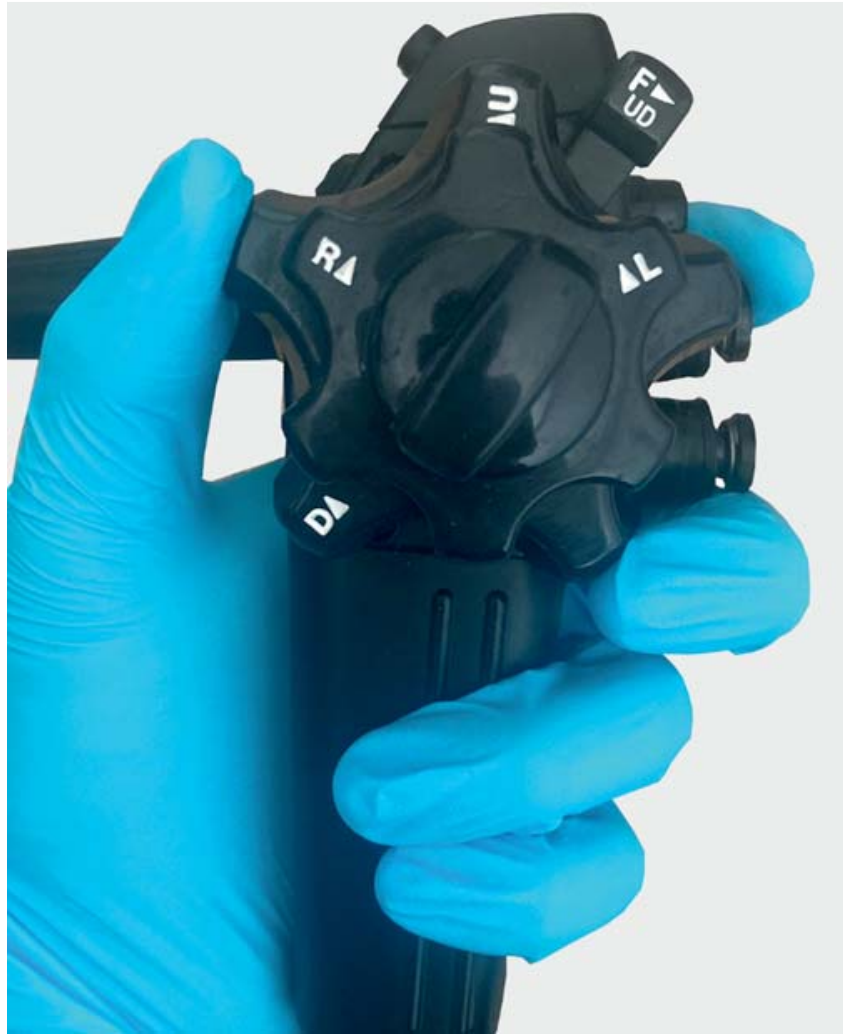

Fig. 2 The handle must be held in the palm of the left hand, with a middle finger positioned to accompany the crutches (especially the up/down crutch).

because the tube is much less constrained in these locations than in the right angle, for example. You need to take the time needed in the sigmoid not to create loops and to use without moderation the shortening technique (colonic shortening technique) [5], the underwater technique [6], and the compression of the sigmoid or the transverse to reach the area of the lesion with a tube that is as short as possible and which is free, stable, and responds to your forward and backward movements. Otherwise, change to a stiffer tube, if possible (i.e. switch from Fuji TM740 or Olympus PCF 190 Ti to PCF 190 L) and compress right away or change position to shorten the transverse (turn the patient to the right while crossing the transverse and put him or her back once in the right colon). Recently, rigidizing overtube has been described to prevent loop formation during colonoscopy [7]. In any case, if you already have difficulty seeing the lesion because of an unstable tube, it is unlikely that the dissection will go well. Once the lesion is reached, holding the endoscope to allow lateral movements around and under the lesion is very important. The right hand must be free to hold the knife or on the tube to accompany the twisting movements of the endoscope. The handle must be held in the palm of the left hand, with a middle finger positioned to accompany the crutches (especially the up/down crutch). This frees the thumb of the left hand to make adjustment movements with the right/left crutch for optimal movements during resection ( $>$ Fig. 2 ). 


\section{Marking the lesion}

In locations where visualization of lesion edges can be difficult (esophagus, stomach), it is imperative to mark the margins of the lesion by putting small coagulation dots a few millimeters (ideally $5 \mathrm{~mm}$ ) around the lesion. In the colon and rectum, it is often easier to see the limits of the lesion because the differences from normal mucosa are clear and experienced operators can skip this step.Indeed, marking the lesion can generate holes within the mucosa, and thus, be responsible for a leakage of the lifting solution, leading to a decreased quality of lifting. To limit this leakage, soft coagulation current is the reference setting for this step (soft coagulation effect 6.5 for ERBE VIO3 or effect 1, $30 \mathrm{w}$ for $\mathrm{VIO} 2$ ). Other currents can create deep lesions of the mucosae through which lifting solution can leak. This step is probably essential for beginners. In the colon, the marking must be very superficial with a foam-tipped tool (hot forceps for example) so as not to create leakage areas for the liquid by piercing the mucosa with the coagulation dots.

\section{Submucosal injection}

\section{Mistakes not to be made}

It is important to have a good understanding of the injection, which is a key element of the procedure, in order to enlarge the submucosal space and thus work safely. The injection must be submucosal and not in another layer. Two frequent mistakes can hinder the procedure: injection that is too superficial or too deep. When the injection is too superficial, in the mucosal layer or in the muscularis mucosae, the mechanical dissection caused by the instillation of liquid into this poorly elastic tissue leads to lesions in the small vessels of the mucosa and almost systematically leads to formation of a very superficial hematoma, which tends to persist until the end of the procedure ( Fig.3). The problem with this hematoma is that it prevents good visualization of the lesion margins and it is sometimes necessary to pass through it to incise the mucosa, which leads to a flow of blood polluting the visual field. Even in the hands of experienced endoscopists, it is common for these small hematomas form, so it is necessary to know how to recognize them immediately in order to stop the injection. When a large hematoma has nevertheless formed, it is necessary to try to reach the submucosa by going below the hematoma to find a clean plan that does not bleed all the time, which will facilitate continuation of the procedure.

On the contrary, when the injection is too deep, the liquid will spread either intraperitoneally or sometimes in the sub-serous layer, resulting in less clean and less bluish lifting. Once again, it is important to be able to recognize this and not to start incising. The submucosa is not elevated, so the risk of muscle injury during the incision is not negligible.

\section{How to inject in the right plane?}

The objective of the injection is to fill the submucosal plane with a current-conducting liquid, i.e., containing ions, which pure water does not allow. To obtain the first lift with the needle, it is necessary to prick deeply and to inject very gently by

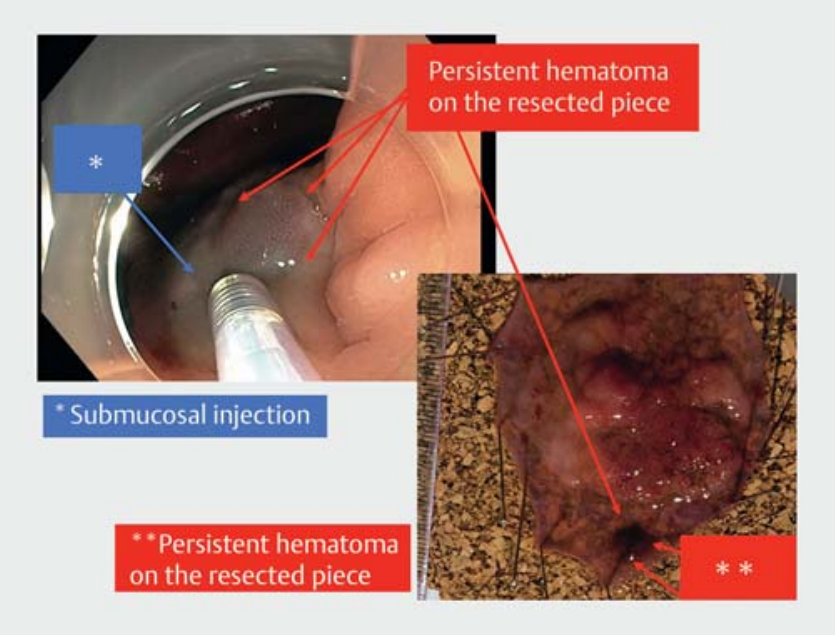

Fig. 3 An injection that is too superficial leads to a hematoma.

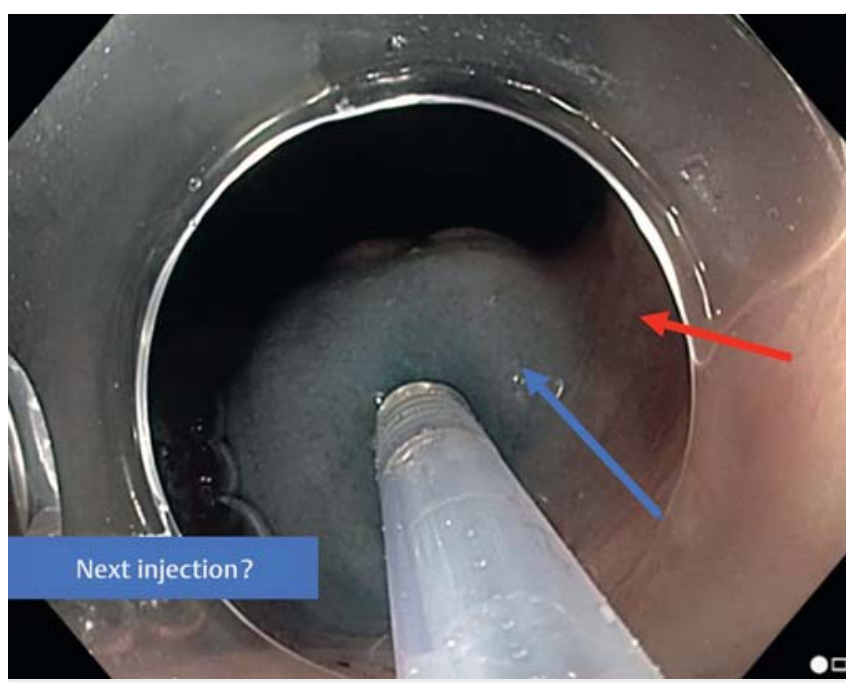

- Fig. 4 To extend the area of submucosal injection, it is better to reinject at the edge of the already injected area (blue arrow) instead of injecting in an area not already injected (red arrow).

gradually withdrawing the needle until a bluish fish bladder bubble appears, reflecting a submucosal injection. Another option is possible, without a needle, with high-pressure knife injectors, such as the Hybrid Knife (Erbe, Tuebingen, Germany) or the Nestis Enki II (Nestis, Lyon, France), which allow the pressurized water jet to pass through the mucosa and fill the submucosa directly $[8,9]$.

\section{How to widen the injected area?}

Once the injection plane has been found with a nice bluish bubble, it is quicker to reinject into the edge of the raised and bluish area to gradually widen the already raised plane rather than pricking again into the non-raised mucosa, which risks injecting too shallowly or too deeply again ( $\triangleright$ Fig. 4 ). 


\section{The good needle}

The injection needle must be carefully chosen so that it is a good compromise between a gauge allowing easy penetration through the mucosa (the smaller the gauge of the needle is, the easier it is) and having a flow rate large enough to inject hard and relatively quickly. In our team in Lyon, France, we use a $25 \mathrm{G}$ needle with a length of $4 \mathrm{~mm}$, which offered us the best compromise after several trials. It is also important to choose an injection needle with a metallic distal sheath that allows the needle to openin theaxis, whatever theposition of theendoscope, including retrovision. Indeed, if the plastic sheath is notrigid enough and the endoscopenotperfectlystraight, theneedlecan piercethesheath, which also increases the risk ofdamaging the endoscope.

\section{Helpful injection}

It is very important to see the injection as a key element requiring anticipatory work to obtain a very high bubble facing the endoscope at the location of the future incision. An injection perpendicular to the mucosal plane facilitates cutting deeper into the submucosa, which has several advantages that we will describe later, by reducing the risk of bleeding and by contributing to part of the trimming, favoring the wide opening of the submucosal plane ( $\boldsymbol{F i g . 5}$ ). The choice of injection site is crucial. Unlike with mucosectomy, it is not lifting the lesion that is desirable, but lifting the cutting plane away from the lesion. As a general rule, the injection should be made $1 \mathrm{~cm}$ away from the lesion to have the most elevated area $0.5 \mathrm{~cm}$ from the lesion to begin the mucosal incision. If the lesion is on a fold, it is sometimes necessary to inject even further away $(15 \mathrm{~mm}$ before the lesion) to facilitate positioning of the endoscope in the submucosa and then to allow the fold to be crossed. If the injection is started too close to the fold and the lesion is large, it can sometimes be difficult to perform effective trimming, and therefore, to gain access to the submucosal layer. As soon as the assistant stops injecting, the liquid leaks through the injection point and it is, therefore, necessary to hurry to change the tool because the beautiful bubble will collapse irremediably, because of the submucosal diffusion of the injection product and the leakage of the liquid through the injection port. Therefore, quick exchange of the injection tool to quickly start the incision is also a key element.

\section{Maintaining lift}

There are two main strategies for maintaining the submucosal plane lift: 1) frequently reinjecting the liquid into the submucosa (facilitated by the injector knives which avoid changing tools continuously to reinject); and 2) using lifting solutions that have a prolonged effect.

\section{Repeated reinjection}

The best way to refill the submucosa in the working area is to frequently inject exactly in the working area to obtain optimal lifting of the area to be cut while reducing risk of diffusion. These volume-limited but frequent reinjection strategies were initially not time-saving when it was necessary to change the tool to inject and to cut. With the availability of injector knives,

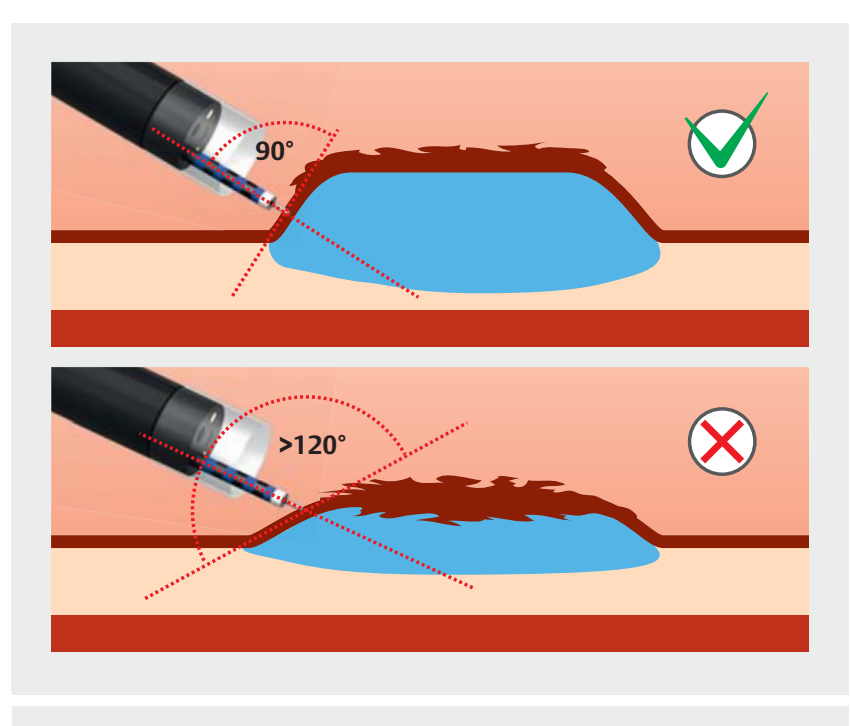

Fig. 5 A perpendicular incision is better to allow a deep incision.

which offer both cutting and injection function (with or without high pressure), this problem has been partially solved because the same tool can alternate between injection and section phases. It is important to note that knives with injection without high pressure do not allow the first injection to be made safely through the mucosa. Thus, it is essential to do the first injection with the needle to find the appropriate plane. The knife then facilitates repeated reinjections to amplify the lift in the working area.

\section{Prolonged lifting solution}

The other option to maintain a high-quality and durable lifting effect is to inject a solution with limited diffusion, and with surface-active properties enabling a high and stable uplift and not lateral extension.

Much research has been done on viscous solutions and there are now several high-viscosity products available, such as hyaluronic acid [10] or poloxamers, which offer interesting properties of variable viscosity according to temperature [11-13]. These products are efficient but expensive. Also, use of viscous solution can result in some bubbles during cutting, thus hindering visibility during dissection. Less viscous but also less expensive alternatives have been tested, such as hydroxyethylstarch or glycerol mixtures $[8,13,14]$, to take advantage of the benefits of reduced diffusion solutions while reducing the cost.

\section{Combined approach}

There is no study demonstrating which is the most effective strategy for repeated reinjections-low-viscosity fluids or less frequent injections of macromolecular solution-because today use of injector knives is common. However, we demonstrated that combining repeated (high-pressure) injection of viscous solutes such as the hyaluronate was more effective ( $25 \%$ speed gain) compared to repeated reinjection of saline solution with the Nestis knife [13]. Another study demonstrated the same benefit by repeatedly injecting less viscous solutions, such as hydroxyethylstarch, compared to the injection of saline with a 
nearly $40 \%$ speed gain with the Hybrid Knife [8]. It is important to be familiar with the equipment you are working with because the roller pump systems used for the Dual Knife Jet (Olympus), Flush Knife (FujifilMm), Splash M Knife (Pentax), and the Orise Proknife (Boston) are not dedicated to the injection of viscous solutions, although experience shows that use of low-viscous solutions, such as glycerol or hydroxyethylstarch, may be possible.

Another question that is often asked is how much blue dye should be added to the liquid. It seems that the concentration needed has decreased as experience with the procedure has progressed. Now, we add only a few drops of dye to color the space. Indeed, it is important to get used to working with a low dose of dye to better see the vessels or muscle in the depth of the submucosa. If the dye is too strong, there is a risk of involuntarily sectioning a vessel or muscle because the contrast between the dark blue submucosa and the vessel is reduced.

To conclude this section of the review, injection is a key step in the procedure that should not be underestimated. It must be considered and integrated into the overall strategy. To maintain uplift, there are unavoidable tools, such as reinjector knives and solutions with a longer half-life. Nevertheless, the uplift gradually decreases and it is therefore imperative not to inject too widely from the outset, but only in areas where one is going to work in the few minutes following the injection.

\section{Mucosal incision}

\section{Mistakes not to be made}

The incision is the step in which the mucosa is cut to access to the submucosal layer. This incision is made at a distance from the lesion to maintain a sufficient margin to reach the $\mathrm{R} 0$ resection. However, the sooner the deep submucosa is accessed, the fewer vessels will be encountered, and the less hemorrhagic oozing will complicate visibility of the submucosal plane. When starting out, it is often difficult to apply significant pressure on the knife from the outset to access the deep submucosa with the first blow, because apprehension about perforating makes us hold the knife back. However, this complicates the procedure if the tip of the knife is not yet in the deep submucosa but in the muscularis mucosa, where many small vessels divide. Another important point is to take sufficient margin around the lesion. Indeed, when we start, we are often obliged to go back several times to incise the mucosa sufficiently deeply, resulting in thermal coagulation lesions at the margin, which tends to threaten the $\mathrm{R} 0$ result of the resection. Make sure to take a 5-mm margin around the lesion so as not to lose the R0 status on a small trajectory error or a difficult incision due to bleeding or a too shy initial incision.

Thus, mucosal incision is probably the most important step:

1. It determines the R0 resection that will no longer be reco-

verable once the incision has been made.

2. It gives access to the submucosal layer more or less quickly and efficiently.

3. It is one of the rare blind and therefore risky phases of the procedure where the tip of the knife is not visible.

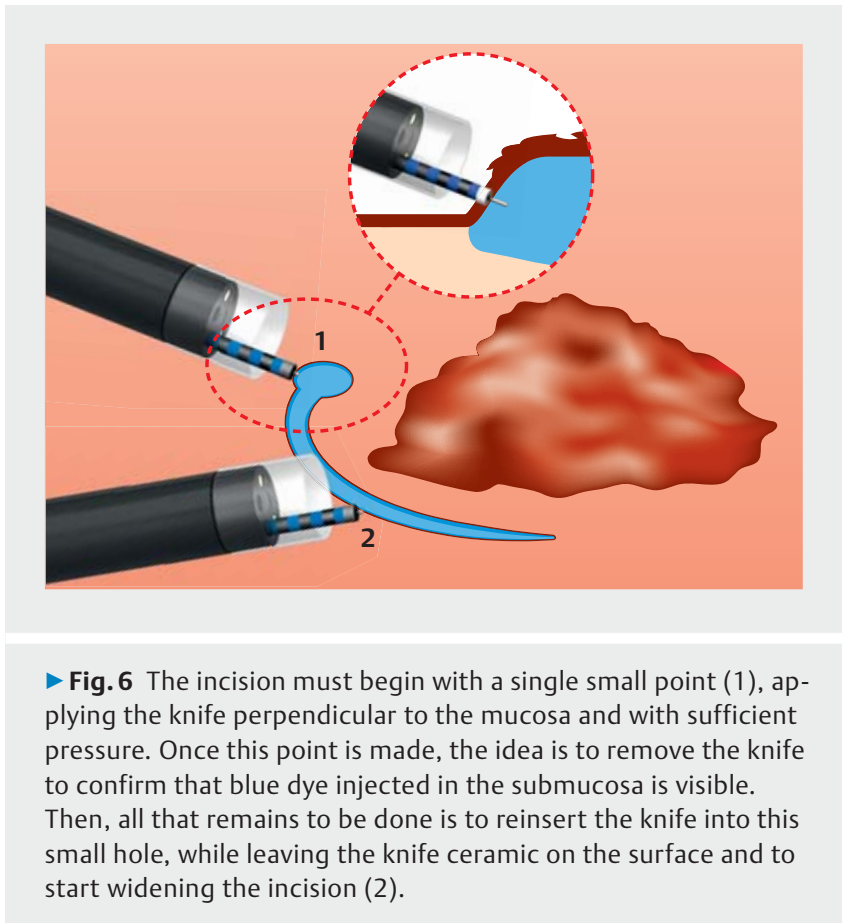

The use of an Endocut I current (settings 2-2-2 ERBE VIO3 or $\mathrm{VIO} 2$ ) is the rule for this step.

The incision must begin with a single small point, applying the knife perpendicular to the mucosa and with sufficient pressure. With experience, one even feels a certain jerk corresponding to the passage of the knife blade through the submucosa through the muscularis mucosae. Once this point is made, the idea is to remove the knife to check to see the blue dye injected in the submucosa. If it is visible, then the incision is deep enough at this point. All that remains to be done is to reinsert the knife into this small hole while leaving the knife ceramic on the surface and start widening the incision. This small hole allows you to calibrate the depth of the incision to the correct level and move from this point to the right and left to make a nice incision, but with sufficient depth calibrated ( Fig.6).

\section{Key step between incision and dissection: trimming.}

This is surely the key moment of the procedure: Not letting an area that has just been incised be emptied while performing a small complementary dissection at the same place to widen this area and not to prevent wider submucosal access a little later. Indeed, any incised area will empty itself of its liquid and become very flat due to the mucous leaks generated. This phenomenon is even more pronounced for beginners, who sometimes take a long time before returning to dissect an incised area (> 20 minutes), whereas the half-life of a mucosal uplift is around 10 minutes with saline solution or glycerol. Therefore, the most effective strategy is to incise small areas, and to pass over the incised submucosa again, dissecting by a few millimeters to widen the incised area and facilitate subsequent access to the submucosa ( $\triangleright$ Fig. 7 ). Therefore, the incision-trimming sequence is surely much more effective than a large incision alone followed by an attempt to dissect an area drained of its 


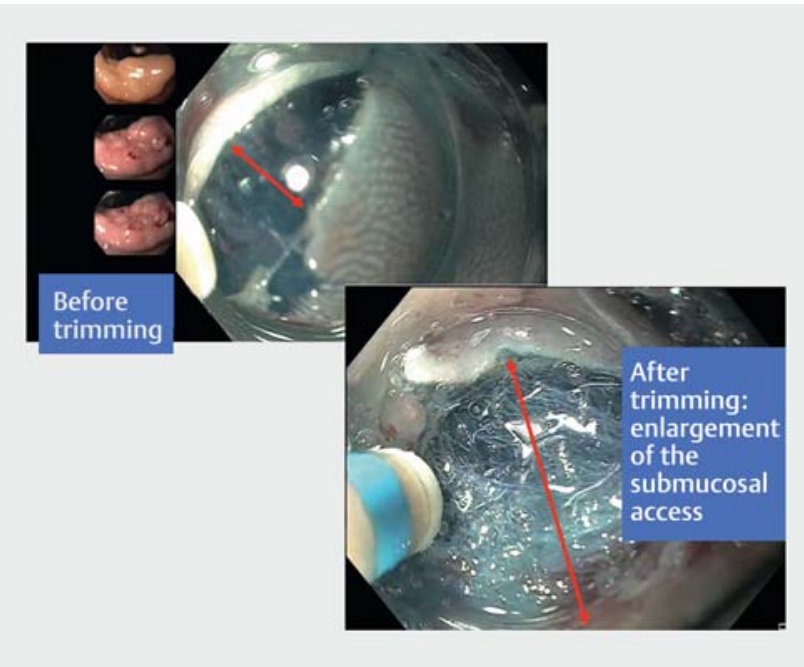

- Fig. 7 Access to the submucosal layer before and after trimming.

submucosal fluid a few minutes later. Besides, the operator, by linking small incision phases with immediate trimming, benefits from an immediate manual memory to immediately return to the correct cutting plane and facilitate this perilous but essential phase. Depending on your experience, it may be a good idea to make an incision of 5 to $10 \mathrm{~mm}$ in length, then interrupt the incision to make the trimming and dissect before continuing the incision and then the trimming alternately by widening the incised area. Well-done trimming is also a key step at the end of the procedure, which will be simplified if the incision has been "cleared" of all the fibers of the superficial muscularis mucosae or submucosa, which can be particularly penalizing at the end of the procedure.

Trimming is performed using a swift coagulation current (effect 5 to 6.5 for VIO 3, effect 2 to 4 ; 40 to $80 \mathrm{~W}$ for VIO 2; depending on the knives). For the past 2 years, expert teams have preferred to perform this step with Endocut current to reduce coagulation artifacts that can hamper the interpretation of margins; however, this very efficient strategy is risky for less expert operators because of an increased risk of bleeding.

\section{Reducing incision size}

We will explore this point in more depth in the dissection section. It is no longer a question of making an incision everywhere at once, but rather, of creating a limited incision to access the submucosal plane without increasing the size of the incised area through which the injected liquid will leak more quickly.

\section{Submucosal dissection}

One of the keys to submucosal dissection is proper exposure of the submucosal plane to dissect it gradually. Initially, the only way to improve this visibility was to turn the patient so that the lesion weighs on the submucosa with the gravity, and thus, widens the submucosal plane.

The setting used during this phase is a swift coagulation current (effect 5 to $6.65 \mathrm{VIO}$ 3, effect 2 to 4 ; 40 to $80 \mathrm{~W}$ VIO 2; de-

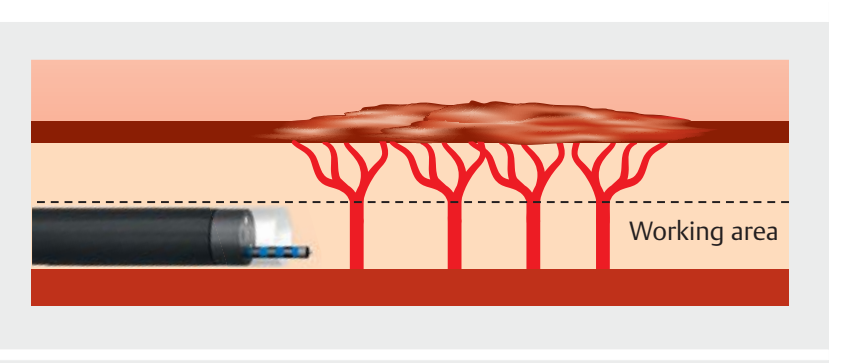

- Fig. 8 It is important to go deep into the submucosal layer to increase the safety margins and to reduce the risk of bleeding by only attacking the large vascular trunks that branch out into the superficial part of the submucosa.

pending on the knives). Here again, the Endocut I current is increasingly used by expert teams in the absence of large vessels because it increases the precision and speed of the procedure.

Whatever the strategy used, it is important to go deep into the submucosal layer ( $\mathbf{F i g . 8}$ ) to increase the safety margins in case of cancer, but also to reduce the risk of bleeding by only attacking the large vascular trunks that branch out into the superficial part of the submucosa.

Since the beginnings of submucosal dissection, several technical elements have been described to improve exposure and thus facilitate the procedure.

\section{Reducing entry surface in the submucosal layer}

The original strategy of performing the circumferential incision before starting the dissection was very difficult. A large incision promotes leakage of fluid, and therefore, quickly and considerably reduces the thickness of the injected submucosal plane. To reduce this phenomenon, several teams were interested in strategies to restrict incision, followed by a phase of dissection under the lesion without the entire circumference being dissected.

\section{Esophageal tunnel strategy}

The esophageal tunnel strategy described in 2013 [15] with limited incisions above and below the lesion allows very good submucosal exposure once the endoscope is introduced into the submucosal plane of the tunnel. Comparative retrospective studies then demonstrated the superiority of this tunneling strategy compared with the standard strategy [16-18]. Moreover, the presence of the endoscope in the tunnel spontaneously creates triangulation by imposing separation of the mucosa from the muscular plane.

\section{Pocket creation method in the colon}

More recently, tunneling strategies also have been described in the rectum, and an adaptation of this technique also has been carried out in the colon with the pocket creation method [19]. These techniques all have the same objective: to limit leakage with a small incision and to take advantage of the submucosal plane to stabilize the endoscope, which is held in the tunnel or pouch, and to improve submucosal exposure, because the endoscope mechanically creates a triangulation. 
These limited incision strategies are very useful in the absence of traction, but we will see that they have been called into question with the availability of traction techniques that reduce the problem of submucosal exposure.

\section{Traction systems}

There are several traction systems:

1. Clip and line traction $[20,21]$ : a wire is placed parallel to the endoscope on a clip inserted in the operating channel. The clip is dropped on the edge of the lesion to be dissected, with the wire coming out through the mouth or the anus. Then, minimal traction is made on the wire to pull the lesion gently and create opposition to the endoscope.

2. Double-clip traction is connected by a dental elastic [2224], a wire loop [25], or a spring. Several variants of these double-clip traction techniques exist, but their purpose is to attach a clip to the lesion's edge, connected for example to a rubber band, and to stretch this rubber band by attaching it with a second clip to the opposite wall of the colon to create a pull.

3. Magnetic traction systems, in which the clip is attached to the lesion and then oriented by a magnetic field external to the patient [26]. These systems are still being evaluated.

4. Robotic endoscopes with two arms, one to cut and the other to pull, which should become common in a few years but are still under development [27].

When using these traction systems, it is necessary to think differently, depending on the location of the lesion to be removed.

\section{In the esophagus}

In the esophagus, the lumen is narrow, and therefore, sufficiently strong traction cannot be applied to the opposite wall of the lesion with the double-clip traction technique. As a result, double-clip traction strategies are not well suited and clip and line traction at the mouth seems to be more suitable. This traction mainly aims to prevent the piece from falling toward the esophagus and twisting the submucosal fibers, which considerably complicates the end of the resection. The description of clip and line traction in esophageal dissection dates from 2014 [25], and we then coupled this traction with the tunneling technique by combining the advantages of these two strategies and demonstrating a coupled benefit [20]. So far, there has been only one randomized study comparing this clip and line strategy with the classical esophageal dissection strategy, demonstrating a procedure time almost halved for similar lesions [28]. For us, the strategy in the esophagus is now well standardized: distal and then proximal incision, creation of a submucosal tunnel between the two, placement of a wire traction at the proximal pole with dental floss or a surgical wire connected to a clip that can be released (and repositioned if possible), and then dissection of the lesion laterally.

\section{In the stomach}

In the stomach, use of these clip and line traction techniques also has reduced risk of perforation in gastric dissection [29] and significantly increased dissection speed [30] for lesions lo- cated in the greater curvature of the stomach. Elastics used in the colon (double-clip traction) can also be a great help in the stomach; however, due to the greater width of the gastric cavity, larger elastics are required.

\section{In the colon}

In the colon, traction is unavoidable. Placement of a traction wire is not obvious because the colonoscope would have to be removed to set it up or slightly complex devices with a line preassembled in the operating channel would be used. Traction techniques that can be set up during the procedure through the operating channel, such as clip and elastic traction [22, 23, 31 ] or the SO clip (double pre-mounted clip with a spring between the two that is inserted into the operating channel [25]), seem simpler to use. It remains to be determined which traction angle is the most efficient and how many traction clips are needed to expose the best, but traction has become unavoidable [22] and randomized trials demonstrating it are being published regularly [32-34]. In an ex vivo colon model, traction increased dissection speed and technical success compared to the pocket creation method, along with decreasing the rate of perforation [33]. A Japanese study showed a reduced procedure time with a traction technique using a clip and thread compared to conventional colonic ESD procedure [34]. Intuitively, it seems that traction at $90^{\circ}$ or perhaps even slightly opposing the endoscope seems to be more effective than traction turning the lesion over ( $\mathbf{F i g . 9}$ ). On the other hand, by facilitating submucosal exposure, traction slightly modified the strategies by reducing the need to make small incisions and to enter in a tunnel. The traction effect is so strong that it is possible to return to larger, even circumferential, incisions because the plane can still be exposed through traction. On the other hand, if a small incision is chosen, traction can make it more difficult to make an incision in the distal part (the edge opposite to the traction). Currently our teams have a standardized strategy with the use of elastic traction ( $\triangleright$ Fig. 10):

1. For lesions smaller than $5 \mathrm{~cm}$, the incision is made circumferentially before traction is applied for progressive dissection from one edge of the lesion to another.

2. For lesions larger than $5 \mathrm{~cm}$ : an incision over one-third or half of the circumference is made distally and then proximally before positioning traction, which will then be combined with a tunneling phase before the end of the lateral incisions and then the residual submucosal edges.

3. For very large lesions or very difficult positioning situations (poor endoscope maneuverability, internal part of the right angle, folds, situation with perpendicular plane, intense fibrosis), the use of several traction systems at the same time may be necessary.

Nevertheless, these types of traction are not adaptive during the procedure as would be robotic independent arms, which are being studied but not yet in use in daily practice. Problems of traction and triangulation should be resolved in the coming years, if robotic endoscopes become accessible to the general public [35] and allow adaptive triangulation at any time, as in 


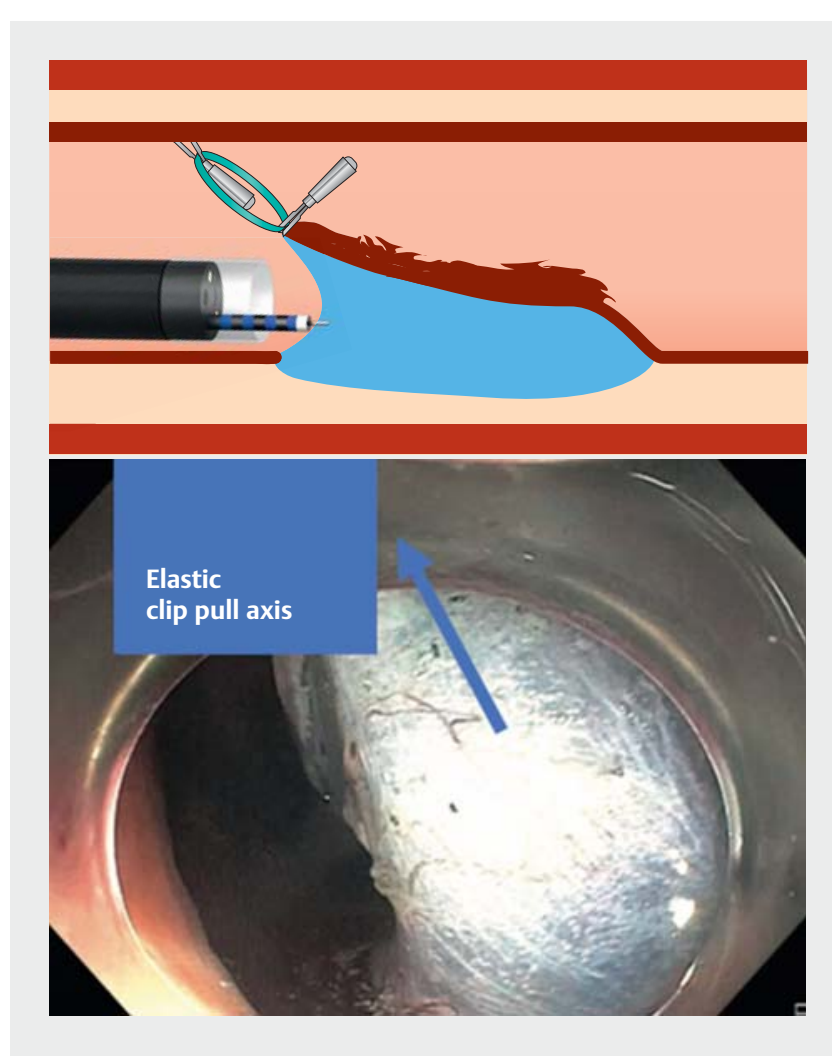

- Fig. 9 Traction at $90^{\circ}$ or perhaps even slightly opposing the endoscope seems to be more effective than traction turning the lesion over.

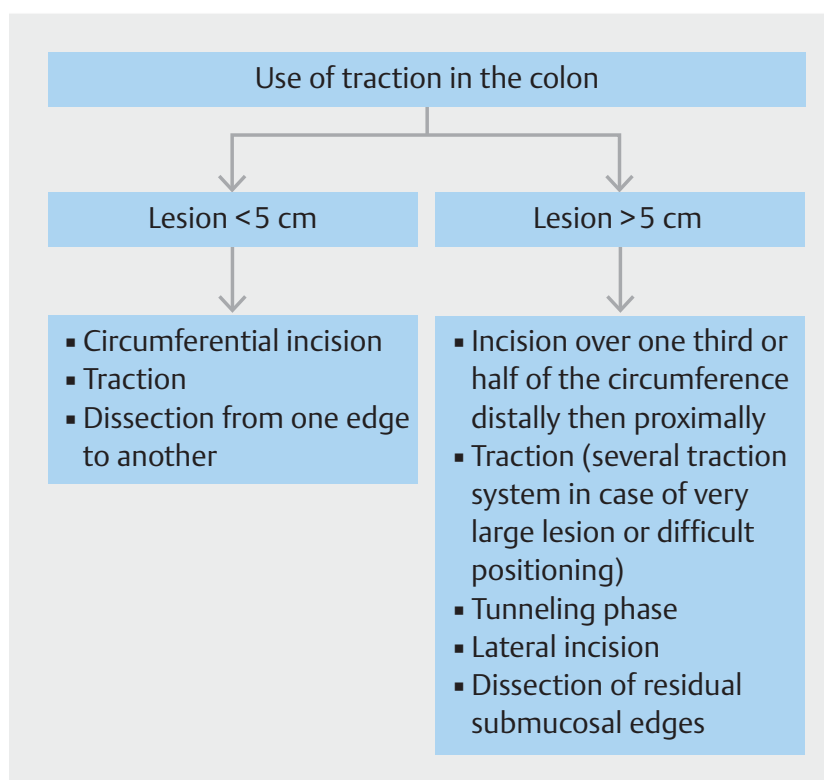

- Fig.10 Our standardized strategy for the use of elastic traction in the colon.

the Hong Kong experiment [36]. While waiting for these robotic systems to finally offer the long-awaited triangulation, lowcost elastic clip traction techniques may facilitate considerably shorter dissection times compared to procedures without traction. Despite the beneficial effect of traction, animal models have demonstrated that combining traction with favorable gravity increases dissection speeds compared to traction with unfavorable gravity. The importance of turning the patient to take advantage of favorable gravity, therefore, remains true despite traction techniques to simplify the procedure and improve speed. On the other hand, changing the patient's position can considerably modify the maneuverability of the endoscope, for better or worse. Our teams now prefer a straight, uncurled, and maneuverable endoscope with unfavorable gravity to a curled endoscope, which is unresponsive but with favorable gravity.

\section{Complications}

Management of bleeding and perforation is an important part of ESD procedure, a source of anxiety, and can threaten the ultimate success of the procedure if bleeding episodes multiply or are difficult to manage ( $\triangleright$ Fig. 11 ).

\section{Management of bleeding}

Bleeding is rarely serious in ESD, although it can be very impressive on the screen. Therefore, it is important to put things into perspective when bleeding occurs and not to panic. In certain areas such as the lower rectum, bleeding is more frequent, and it is certainly more effective to coagulate the visible vessels to prevent bleeding rather than repeatedly treating bleeding triggered by the cut. In general, when a vessel is well identified in the submucosa, it is better to change the tool (coagulation forceps) to preventively coagulate it. With experience, one learns that some vessels can be coagulated with the tip of the knife, but it takes time to find the right compromise.

When the bleeding is triggered and abundant, the keys are to expose the bleeding point well and not to try to coagulate randomly in the blood pool. To do this, use the cap to tamponade the area by pressing on the vessel and backing off gently to see the bleeding point clearly. If the bleeding is too profuse and the blood accumulates in the resection area, do not hesitate to turn the patient so that gravity allows the blood to flow elsewhere.

A new technology may soon help us manage this bleeding with the RDI mode (Olympus, Tokyo, Japan), which allows the active bleeding point (bright red) to be identified within the blood sheet (orange appearance) by contrasting the reds. This mode has already demonstrated its considerable contribution to help the operator to control bleeding (significantly shorter clotting time) but also to reduce the practitioner anxiety (fewer eye movements, less tachycardia) $[37,38]$.

To control bleeding, thermal hemostasis techniques (hot forceps) are preferable because they do not interfere with continuation of dissection, unlike clips, which can close the submucosal plane or generate an obstacle to continuing dissection.

\section{Management of perforation}

We are all a little afraid of perforation, even after a few dozen experiences with it, but risk of having to operate on the patient 


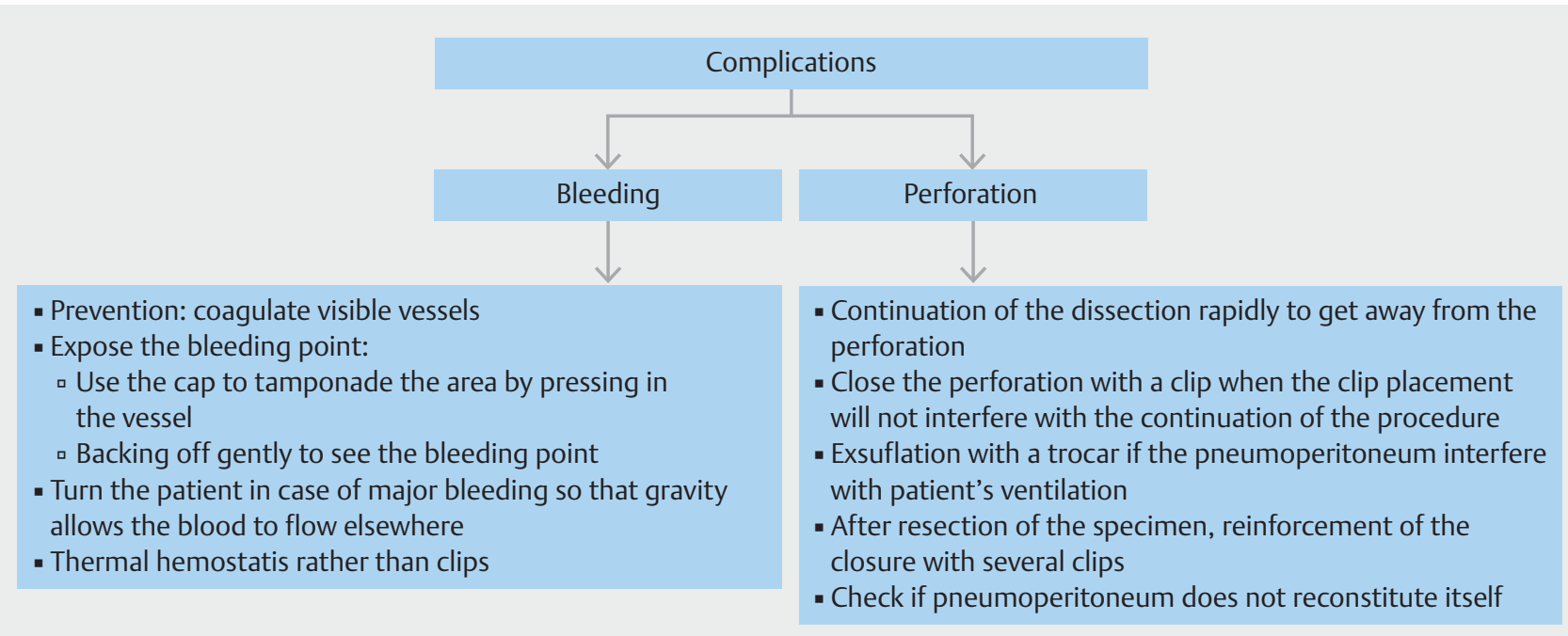

- Fig. 11 Management of the two main complications of ESD: bleeding and perforation.

in the aftermath of this complication is low if you manage to relax and close a perforation tightly. If necessary, do not hesitate to transfer the procedure to the hands of a less anxious or tired colleague. Here again, things have changed a lot and we continue dissection despite a small hole, not by leaving, but continuing to dissect the area rapidly to get away from the perforation. Then we close when we think that placement of the clip will no longer interfere with continuing dissection. If the pneumoperitoneum interferes with the patient's ventilation, then it is exsufflated with a trocar and the procedure is continued. On the other hand, once the area with the hole is accessible and the specimen has been resected, the closure is reinforced with several other clips, checking afterwards that the pneumoperitoneum does not reconstitute itself when the colon is insufflated. Data to validate these attitudes are lacking [39], but in our experience, currently awaiting publication, more than $95 \%$ of perforations of less than $5 \mathrm{~mm}$ are managed endoscopically without subsequent recourse to surgery. For large perforations (>5 mm), the clips must first be positioned on either side of the perforation to bring the edges together (like a zipper) before then effectively catching the perforated area.

\section{Postoperative management}

European Society of Gastrointestinal Endoscopy guidelines do not include any recommendation on postoperative management of patients following ESD [40]. In our group, patients are hospitalized until the day after the procedure. The evening after the procedure, they can only drink water without any food. The day after that, they can eat a normal meal and if they do not have any pain, blood in stools, or fever with normal bowel movement, they can leave the hospital. We do not give antibiotics systematically. Blood tests the day after the procedure are not systematically performed. Of course, this management must be adapted to the patient history (anti-coagulant treatment with the need of a bridging therapy for example) or the procedure itself (major bleeding, extended perforation, etc).

\section{Conclusions}

The ESD technique has evolved greatly and resection strategies have changed dramatically. Injection must always be performed very carefully and anticipated by injecting the area of interest to allow a dome shape and not a plateau. Injector knives make it easier to reinject the area of interest without changing the tool and make expensive solutions with prolonged lifting less necessary. The incision must be frank and deep, starting from a small point at the right depth and alternating incision and trimming to really open the space and access the submucosa. Small incisions followed immediately by dissection are still appropriate, even though small incisions to access a tunnel or other pockets are less necessary with use of traction. Today, the ideal strategy almost always includes a traction technique adapted to the organ, to better expose the plane and accelerate dissection speeds, while reducing the risk of perforation. Bleeding and perforation are rare events and should be kept in perspective because, as long as you stay calm, endoscopic management can cure the patient in over $95 \%$ of cases. Comparative scientific data are still needed to show the benefits of optimized ESD compared to standard techniques and to quantify the gains in efficiency and safety of these optimizations.

\section{Competing interests}

Drs. Pioche, Jacques, Legros, and Rivory are consultants for Olympus, Norgine, Fuji, and Erbe.

References

[1] Fuccio L, Repici A, Hassan C et al. Why attempt en bloc resection of non-pedunculated colorectal adenomas? A systematic review of the prevalence of superficial submucosal invasive cancer after endoscopic submucosal dissection. Gut 2018; 67: 1464-1474 
[2] Baniya R, Upadhaya S, Khan J et al. Carbon Dioxide versus air insufflation in gastric endoscopic submucosal dissection: a systematic review and meta-analysis of randomized controlled trials. Clin Endosc 2017; 50: 464-472

[3] Maeda Y, Hirasawa D, Fujita $\mathrm{N}$ et al. Carbon dioxide insufflation in esophageal endoscopic submucosal dissection reduces mediastinal emphysema: A randomized, double-blind, controlled trial. World J Gastroenterol 2016; 22: 7373-7382

[4] Kim SY, Chung J-W, Kim JH et al. Carbon dioxide insufflation during endoscopic resection of large colorectal polyps can reduce post-procedure abdominal pain: A prospective, double-blind, randomized controlled trial. United European Gastroenterol J 2018; 6: 1089-1098

[5] Lee S-H, Park Y-K, Lee D-J et al. Colonoscopy procedural skills and training for new beginners. World J Gastroenterol 2014; 20: 16984 16995

[6] Lesne A, Rouquette O, Touzet S et al. Adenoma detection with bluewater infusion colonoscopy: a randomized trial. Endoscopy 2017; 49: 765-775

[7] Wei MT, Hwang JH, Watson RR et al. Novel rigidizing overtube for colonoscope stabilization and loop prevention (with video). Gastrointest Endosc 2021; 93: 740-749

[8] Jacques J, Sautereau D, Carrier P et al. High-pressure injection of glycerol with HybridKnife for ESD is feasible and increases the ease and speed of the procedure: an in vivo study in pigs and first use in human. Surg Endosc 2015; 29: 3382-3385

[9] Pioche M, Lépilliez V, Ciocîrlan M et al. Endoscopic submucosal dissection with the Nestis ${ }^{\circledR}$ jet injector system with a bifunctional catheter: first prospective clinical trial (NCT: 2012-A00272-41). Surg Endosc 2016; 30: 5140-5146

[10] Córdova H, Cuatrecasas M, García-Rodríguez A et al. Successful outcomes of a new combined solution of hyaluronic acid, chondroitin sulfate and poloxamer 407 for submucosal injection: animal survival study. Endosc Int Open 2019; 7: E576-E582

[11] Giannino V, Salandin L, Macelloni C et al. Evaluation of Eleview ${ }^{\circledR}$ Bioadhesive Properties and Cushion-Forming Ability. Polymers; Basel: 2020: 12

[12] Fujishiro M, Yahagi N, Kashimura K et al. Different mixtures of sodium hyaluronate and their ability to create submucosal fluid cushions for endoscopic mucosal resection. Endoscopy 2004; 36: 584-589

[13] Pioche M, Ciocirlan M, Lépilliez V et al. High-pressure jet injection of viscous solutions for endoscopic submucosal dissection: a study on ex vivo pig stomachs. Surg Endosc 2014; 28: 1742-1747

[14] Tall ML, Salmon D, Diouf E et al. Aseptic process validation and stability study of an injectable preparation of fructose (5\%)-glycerol (10\%) as part of a hospital clinical research program on endoscopic curative treatment for early epithelial neoplastic lesions of the gastrointestinal tract. Ann Pharm Fr 2015; 73: 139-149

[15] Pioche M, Mais L, Guillaud O et al. Endoscopic submucosal tunnel dissection for large esophageal neoplastic lesions. Endoscopy 2013; 45: 1032-1034

[16] Zhang T, Zhang H, Zhong F et al. Efficacy of endoscopic submucosal tunnel dissection versus endoscopic submucosal dissection for superficial esophageal neoplastic lesions: a systematic review and metaanalysis. Surg Endosc 2021; 35: 52-62

[17] Li P, Ma B, Gong S et al. Endoscopic submucosal tunnel dissection for superficial esophageal neoplastic lesions: a meta-analysis. Surg Endosc 2020; 34: 1214-1223

[18] Huang R, Cai H, Zhao X et al. Efficacy and safety of endoscopic submucosal tunnel dissection for superficial esophageal squamous cell carcinoma: a propensity score matching analysis. Gastrointest Endosc 2017; 86: 831-838

[19] Sakamoto H, Hayashi Y, Miura Y et al. Pocket-creation method facilitates endoscopic submucosal dissection of colorectal laterally spreading tumors, non-granular type. Endosc Int Open 2017; 5 : E123-E129

[20] Jacques ], Geyl S, Carrier P et al. A combination of the clip-with-line method and the tunnel technique during esophageal endoscopic submucosal dissection: a technical solution? Endoscopy 2015; 47 : (Suppl. 01): E307-E308

[21] Yamasaki Y, Takeuchi Y, Uedo N et al. Traction-assisted colonic endoscopic submucosal dissection using clip and line: a feasibility study. Endosc Int Open 2016; 4: E51-E55

[22] Jacques ], Charissoux A, Bordillon P et al. High proficiency of colonic endoscopic submucosal dissection in Europe thanks to countertraction strategy using a double clip and rubber band. Endosc Int Open 2019; 7: E1166-E1174

[23] Faller J, Jacques J, Oung B et al. Endoscopic submucosal dissection with double clip and rubber band traction for residual or locally recurrent colonic lesions after previous endoscopic mucosal resection. Endoscopy 2020; 52: 383-388

[24] Oung B, Rivory ], Chabrun E et al. ESD with double clips and rubber band traction of neoplastic lesions developed in the appendiceal orifice is effective and safe. Endoscopy International Open 2020; 08: E388-E395

[25] Nagata M, Fujikawa T, Munakata H. Comparing a conventional and a spring-and-loop with clip traction method of endoscopic submucosal dissection for superficial gastric neoplasms: a randomized controlled trial (with videos). Gastrointest Endosc 2020; 93: 1097-1109

[26] Matsuzaki I, Hattori M, Hirose K et al. Magnetic anchor-guided endoscopic submucosal dissection for gastric lesions (with video). Gastrointest Endosc 2018; 87: 1576-1580

[27] Turiani Hourneaux de Moura D, Aihara H, Jirapinyo P et al. Robot-assisted endoscopic submucosal dissection versus conventional ESD for colorectal lesions: outcomes of a randomized pilot study in endoscopists without prior ESD experience (with video). Gastrointest Endosc 2019; 90: 290-298

[28] Oyama T. Counter traction makes endoscopic submucosal dissection easier. Clin Endosc 2012; 45: 375-378

[29] Koike Y, Hirasawa D, Fujita N et al. Usefulness of the thread-traction method in esophageal endoscopic submucosal dissection: randomized controlled trial. Dig Endosc 2015; 27: 303-309

[30] Yoshida M, Takizawa K, Suzuki S et al. Conventional versus tractionassisted endoscopic submucosal dissection for gastric neoplasms: a multicenter, randomized controlled trial (with video). Gastrointest Endosc 2018; 87: 1231-1240

[31] Oung B, Chabrun E, Subtil C et al. Traction strategy with clips and rubber band allows complete en bloc endoscopic submucosal dissection of sessile serrated adenoma/polyp invading the site of previous appendectomy. Endoscopy 2019; 51: E166-E168

[32] Ritsuno H, Sakamoto N, Osada T et al. Prospective clinical trial of traction device-assisted endoscopic submucosal dissection of large superficial colorectal tumors using the S-O clip. Surg Endosc 2014; 28: 3143-3149

[33] Albouys ], Dahan M, Lepetit H et al. Double-clip traction could be superior to the pocket-creation method with cylindrical cap for colonic ESD: a randomized study in an ex vivo model. Surg Endosc 2021; 35 : 1482-1491

[34] Yamasaki Y, Takeuchi Y, Uedo $\mathrm{N}$ et al. Efficacy of traction-assisted colorectal endoscopic submucosal dissection using a clip-and-thread technique: A prospective randomized study. Dig Endosc 2018; 30 : 467-476

[35] Takeshita N, Ho KY, Phee S] et al. Feasibility of performing esophagea endoscopic submucosal dissection using master and slave transluminal endoscopic robot. Endoscopy 2017; 49: E27-E28

[36] Chiu PWY, Ho KY, Phee S]. Colonic endoscopic submucosal dissection using a novel robotic system (with video). Gastrointest Endosc 2020; 93: 1172-1177 
[37] Maehata T, Fujimoto A, Uraoka T et al. Efficacy of a new imageenhancement technique for achieving hemostasis in endoscopic submucosal dissection. Gastrointest Endosc 2020; 92: 667-674

[38] Fujimoto A, Saito Y, Abe $S$ et al. Haemostasis treatment using dual red imaging during endoscopic submucosal dissection: a multicentre, open-label, randomised controlled trial. BMJ Open Gastroenterol 2019; 6: e000275
[39] Pioche M, Bertrand G, Rivory J. Endoscopic perforation during EMR corrected or ESD: who should take care of the patient? Endosc Int Open 2018; 6: E313-E314

[40] Pimentel-Nunes P, Dinis-Ribeiro M, Ponchon T et al. Endoscopic submucosal dissection: European Society of Gastrointestinal Endoscopy (ESGE) Guideline. Endoscopy 2015; 47: 829-854 